\title{
WASP-34b: a near-grazing transiting sub-Jupiter-mass exoplanet in a hierarchical triple system ${ }^{\star}$
}

\author{
B. Smalley ${ }^{1}$, D. R. Anderson ${ }^{1}$, A. Collier Cameron ${ }^{2}$, C. Hellier ${ }^{1}$, M. Lendl ${ }^{3}$, P. F. L. Maxted ${ }^{1}$, D. Queloz ${ }^{3}$, \\ A. H. M. J. Triaud ${ }^{3}$, R. G. West ${ }^{4}$, S. J. Bentley ${ }^{1}$, B. Enoch ${ }^{2}$, M. Gillon ${ }^{5,3}$, T. A. Lister ${ }^{6}$, F. Pepe ${ }^{3}$, D. Pollacco , \\ D. Segransan ${ }^{3}$, A. M. S. Smith ${ }^{1}$, J. Southworth ${ }^{1}$, S. Udry ${ }^{3}$, P. J. Wheatley ${ }^{8}$, P. L. Wood ${ }^{1}$, and J. Bento ${ }^{8}$ \\ 1 Astrophysics Group, Keele University, Staffordshire, ST5 5BG, UK \\ e-mail: bs@astro.keele.ac.uk \\ 2 SUPA, School of Physics and Astronomy, University of St. Andrews, North Haugh, Fife, KY16 9SS, UK \\ 3 Observatoire de Genève, Université de Genève, 51 chemin des Maillettes, 1290 Sauverny, Switzerland \\ ${ }^{4}$ Department of Physics and Astronomy, University of Leicester, Leicester, LE1 7RH, UK \\ 5 Institut d'Astrophysique et de Géophysique, Université de Liège, Allée du 6 Août, 17, Bât. B5C, Liège 1, Belgium \\ ${ }^{6}$ Las Cumbres Observatory, 6740 Cortona Dr. Suite 102, Santa Barbara, CA 93117, USA \\ 7 Astrophysics Research Centre, School of Mathematics \& Physics, Queen's University, University Road, Belfast, BT7 1NN, UK \\ 8 Department of Physics, University of Warwick, Coventry CV4 7AL, UK
}

Received 25 October 2010 / Accepted 9 December 2010

\section{ABSTRACT}

\begin{abstract}
We report the discovery of WASP-34b, a sub-Jupiter-mass exoplanet transiting its 10.4-magnitude solar-type host star (1SWASP J110135.89-235138.4; TYC 6636-540-1) every 4.3177 days in a slightly eccentric orbit $(e=0.038 \pm 0.012$ ). We find a

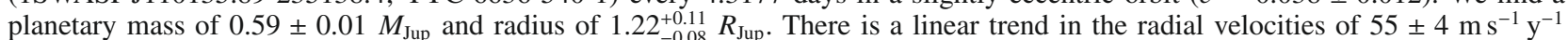
indicating the presence of a long-period third body in the system with a mass $\gtrsim 0.45 M_{\text {Jup }}$ at a distance of $\gtrsim 1.2 \mathrm{AU}$ from the host star. This third-body is either a low-mass star, a white dwarf, or another planet. The transit depth $\left(\left(R_{\mathrm{P}} / R_{*}\right)^{2}=0.0126\right)$ and high impact parameter $(b=0.90)$ suggest that this could be the first known transiting exoplanet expected to undergo grazing transits, but with a confidence of only $\sim 80 \%$.
\end{abstract}

Key words. planets and satellites: general - stars: individual: WASP-34 - techniques: photometric - techniques: spectroscopic techniques: radial velocities

\section{Introduction}

The majority of the known exoplanets have been discovered using the radial velocity technique (Mayor \& Queloz 1995). In recent years, however, an ever increasing number have been discovered as a result of group-based and space-based transit search survey projects. Transiting exoplanets allow parameters such as the mass, radius, and density to be accurately determined, as well as their atmospheric properties to be studied during their transits and occultations (Charbonneau et al. 2005; Southworth 2009; Winn 2009).

The SuperWASP project has robotic observatories in the Canary Islands and South Africa. The wide angle survey is designed to find exoplanets around relatively bright stars in the $V$-magnitude range $9 \sim 13$. A detailed description of the SuperWASP project is given in Pollacco et al. (2006).

In this paper we report the discovery of WASP-34b, an exoplanet in orbit around its $V=10.4$ mag. host star 1SWASP J110135.89-235138.4 in the constellation Crater. We present the WASP-South discovery photometry, together with Euler Telescope photometry and CORALIE radial velocity measurements.

\footnotetext{
* Radial velocity and photometric data are only available in electronic form at the CDS via anonymous ftp to cdsarc.u-strasbg.fr (130.79.128.5) or via http://cdsarc.u-strasbg.fr/viz-bin/qcat?]/A+A/526/A130
}

\section{Observations}

\subsection{WASP-South photometry}

The host star WASP-34 (1SWASP J110135.89-235138.4; CD23 9677; TYC 6636-540-1; GSC 06636-00540) was observed within two WASP-South camera fields during the periods 2006 May 4 to June 20 and 2007 January 4 to June 1, and in 3 fields during the period 2008 January 1 to May 28. A total of 35351 data points were obtained. The pipeline-processed data were de-trended and searched for transits using the methods described in Collier Cameron et al. (2006), yielding a detection of a periodic, transit-like signature with a period of 4.3177 days and a depth of $0.011 \mathrm{mag}$ (Fig. 1).

\subsection{Spectroscopic observations with CORALIE}

Spectroscopic observations were obtained with the CORALIE spectrograph on the Swiss $1.2 \mathrm{~m}$ telescope. The data were processed using the standard pipeline (Baranne et al. 1996; Queloz et al. 2000; Pepe et al. 2002). A total of 24 radial velocity (RV) and line bisector span $\left(V_{\text {span }}\right)$ measurements were made between 2009 December 1 and August 1 (Table 1). The bisector spans are a measure of the asymmetry of the cross-correlation function and, based on our experience, have standard errors of $\approx 2 \sigma_{\mathrm{RV}}$.

The RV measurements show velocity variations with the same period as the transit light curve, but with a relatively 

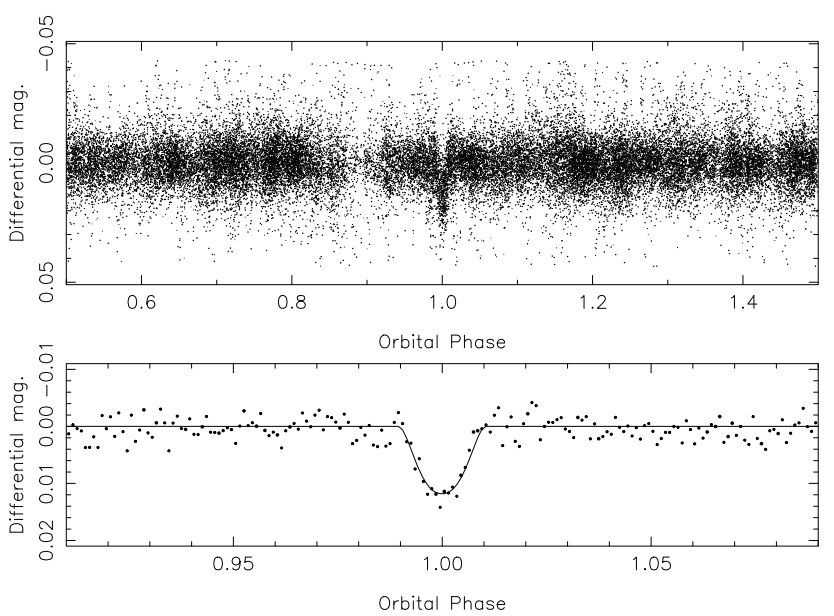

Fig. 1. WASP photometry of WASP-34 folded on the orbital of period of 4.3177 days (upper panel). The lower-panel shows the photometry binned in 0.001 phase steps and centred on the transit. The solid line is best-fit MCMC solution (see Sect. 4)

Table 1. Radial velocity (RV) and line bisector spans $\left(V_{\text {span }}\right)$ measurements for WASP-34 obtained by CORALIE spectra.

\begin{tabular}{lll}
\hline \hline BJD-2 400 000 $(\mathrm{UTC})$ & $\mathrm{RV}\left(\mathrm{km} \mathrm{s}^{-1}\right)$ & $V_{\text {span }}\left(\mathrm{km} \mathrm{s}^{-1}\right)$ \\
\hline 55166.8238 & $49.7903 \pm 0.0044$ & -0.0413 \\
55168.8183 & $49.9372 \pm 0.0043$ & -0.0295 \\
55170.8431 & $49.7923 \pm 0.0042$ & -0.0275 \\
55172.8238 & $49.9253 \pm 0.0046$ & -0.0540 \\
55174.8487 & $49.8141 \pm 0.0041$ & -0.0499 \\
55175.8479 & $49.7973 \pm 0.0039$ & -0.0376 \\
55176.8227 & $49.8806 \pm 0.0042$ & -0.0327 \\
55179.8417 & $49.7888 \pm 0.0041$ & -0.0350 \\
55180.8558 & $49.8611 \pm 0.0041$ & -0.0401 \\
55181.8211 & $49.9414 \pm 0.0042$ & -0.0382 \\
55182.8513 & $49.8765 \pm 0.0049$ & -0.0405 \\
55184.8546 & $49.8432 \pm 0.0044$ & -0.0282 \\
55186.8291 & $49.9058 \pm 0.0046$ & -0.0142 \\
55190.8501 & $49.9152 \pm 0.0045$ & -0.0438 \\
55261.7732 & $49.7686 \pm 0.0049$ & -0.0303 \\
55262.6716 & $49.8191 \pm 0.0041$ & -0.0375 \\
55372.5070 & $49.8731 \pm 0.0050$ & -0.0401 \\
55375.6012 & $49.8797 \pm 0.0070$ & -0.0128 \\
55376.5162 & $49.8956 \pm 0.0080$ & -0.0249 \\
55380.5162 & $49.8922 \pm 0.0048$ & -0.0383 \\
55391.4963 & $49.7631 \pm 0.0053$ & -0.0275 \\
55399.4711 & $49.7695 \pm 0.0048$ & -0.0249 \\
55403.4675 & $49.8159 \pm 0.0049$ & -0.0133 \\
55410.4711 & $49.8913 \pm 0.0049$ & -0.0437 \\
\hline
\end{tabular}

large scatter in the residuals $\left(16.1 \mathrm{~m} \mathrm{~s}^{-1}\right)$ to a circular orbit fit. Analysis of these residuals shows a trend as a function of time of $55 \pm 4 \mathrm{~m} \mathrm{~s}^{-1} \mathrm{y}^{-1}$, with an F-test revealing that this is significant at $>99.9 \%$ level (Fig. 2). The amplitude of the RV variations and the absence of any correlation with RV of the line bisector spans $\left(V_{\text {span }}\right)$ in Fig. 3 indicates that it is highly improbable that the RV variations are due to an unresolved eclipsing binary or chromospheric activity (Queloz et al. 2001).

\subsection{Euler Telescope photometry}

We observed two transits (March 3 and April 12, 2010) of WASP-34b with the $1.2 \mathrm{~m}$ Euler Telescope. All data were taken in the $R_{G}$ band and, owing to the brightness of the target

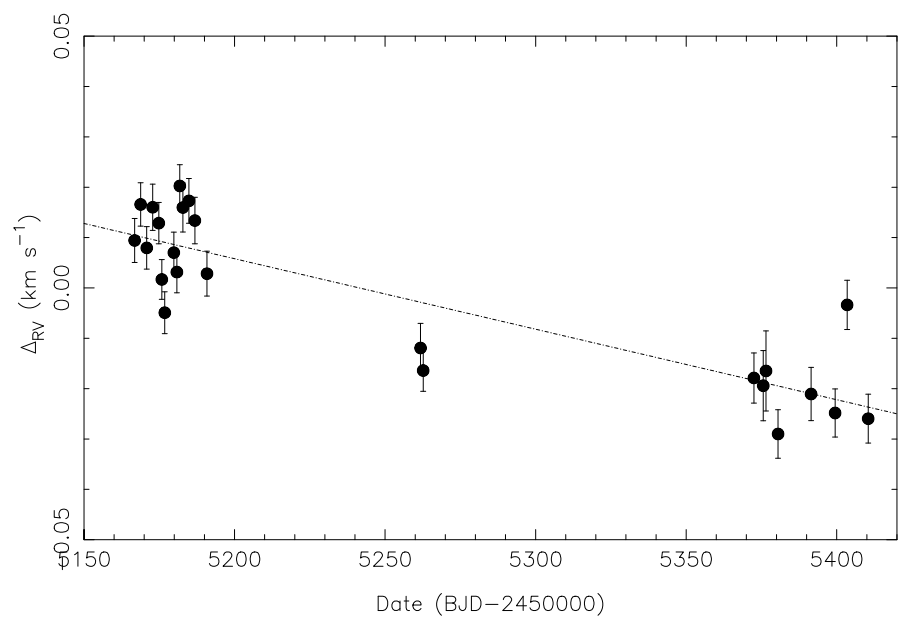

Fig. 2. Residuals to the periodic orbital fit $\left(\Delta_{\mathrm{RV}}\right)$ as a function of time. There is a clear long-term trend in the systemic velocity of $55 \pm 4 \mathrm{~m} \mathrm{~s}^{-1} \mathrm{y}^{-1}$.

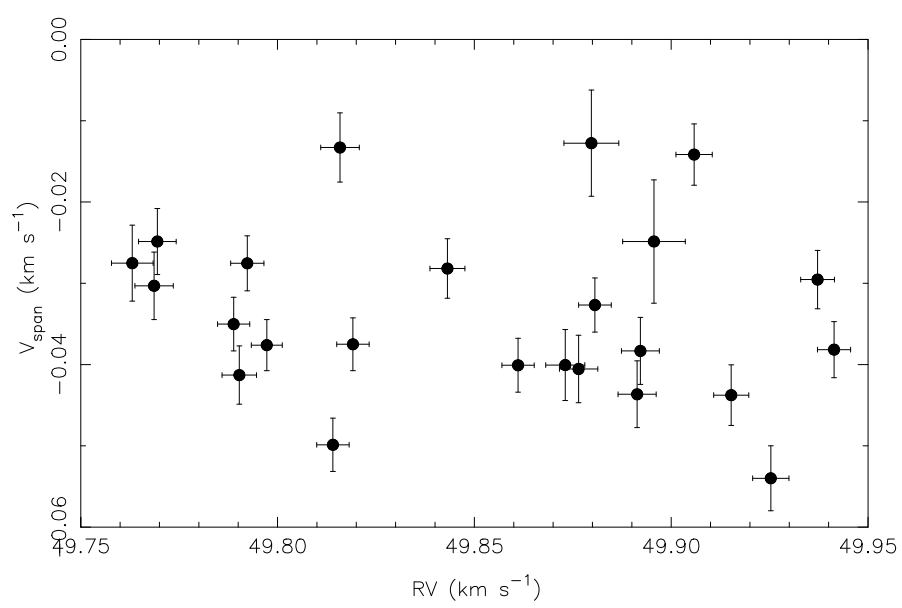

Fig. 3. Line bisectors $\left(V_{\text {span }}\right)$ as a function of RV for WASP-34. Bisector uncertainties of twice the RV uncertainties have been adopted. There is negligible correlation between $V_{\text {span }}$ and the stellar RV.

(mag $R=9.81$ ), with slight defocus. While for the first transit there was no guiding available at the telescope, we made use of a new absolute tracking (ABTR) mode for the second observation. The ABTR performs tracking by computing the location of light sources on each science frame and matching them with a catalogue. This way, the exact position of each image is found and drifts from the nominal position are corrected by adjusting the telescope pointing between the exposures. As a result the target is kept on the same pixel during the whole transit.

The reduction was the same for both transits. After correcting the images for bias, overscan and flatfield, we performed aperture photometry on the data. The flux was extracted for all stars in the field and the final light curve was obtained from differential photometry of the target and a reference source obtained by combining the 3 brightest reference stars. We reached a rms photometric precision of $4.2 \mathrm{~m} \mathrm{mag} \mathrm{min}^{-1}$ for the transit of March 3 and $1.4 \mathrm{~m} \mathrm{mag} \mathrm{min}^{-1}$ for the transit of April 12 (Fig. 4). This improvement can mainly be attributed to the use of the ABTR. As the stars were kept on the same pixels, flat field effects were greatly reduced. 


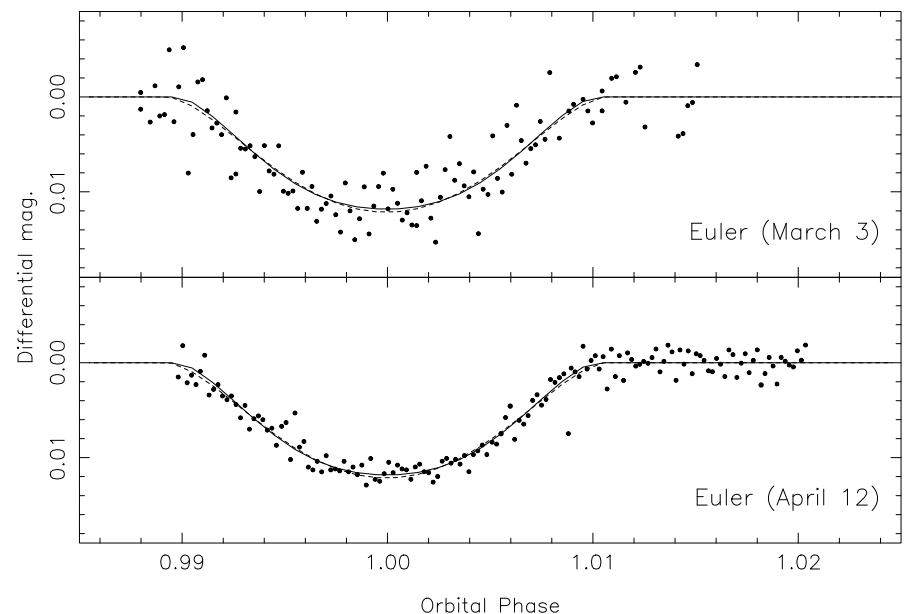

Fig. 4. Euler Telescope transit photometry. The solid line is the best-fit main-sequence-constrained MCMC solution, while the dashed-line is that for the non-main-sequence-constrained solution.

\section{Spectral analysis}

The individual CORALIE spectra of WASP-34 were co-added to produce a single spectrum with an average $S / N$ of around 100:1. The standard pipeline reduction products were used in the analysis.

The analysis was performed using the methods given in Gillon et al. (2009) and Smalley (2005). The $\mathrm{H} \alpha$ line was used to determine the effective temperature $\left(T_{\text {eff }}\right)$, while the Na I D and $\mathrm{Mg}$ I $\mathrm{b}$ lines were used as surface gravity $(\log g)$ diagnostics. The parameters obtained from the analysis are listed in Table 2. The elemental abundances were determined from equivalent width measurements of several unblended lines. A value for microturbulence $\left(\xi_{\mathrm{t}}\right)$ was determined from Fe I using the method of Magain (1984). The quoted error estimates include that given by the uncertainties in $T_{\text {eff }}, \log g$ and $\xi_{\mathrm{t}}$, as well as the scatter due to measurement and atomic data uncertainties.

The projected stellar rotation velocity $(v \sin i)$ was determined by fitting the profiles of several unblended Fe I lines. A value for macroturbulence $\left(v_{\text {mac }}\right)$ of $2.3 \pm 0.3 \mathrm{~km} \mathrm{~s}^{-1}$ was assumed, based on the recent calibration of Bruntt et al. (2010), and an instrumental $F W H M$ of $0.11 \pm 0.01 \AA$, determined from the telluric lines around $6300 \AA$. A best-fitting value of $v \sin i=$ $1.4 \pm 0.6 \mathrm{~km} \mathrm{~s}^{-1}$ was obtained.

The lack of any measurable lithium in the CORALIE spectra implies that WASP-34 is a relatively old star, with an age of $\gtrsim 5$ Gy (Sestito \& Randich 2005). The measured $v \sin i$ of WASP34 implies a rotational period of $P_{\text {rot }} \simeq 34 \pm 15$ days, which yields a gyrochronological age of $\sim 6.7_{-4.5}^{+6.9}$ Gy using the relation of Barnes (2007), which is consistent with the lithium age. In many respects WASP-34 is very similar to the Sun.

\section{Planetary system parameters}

To determine the planetary and orbital parameters the CORALIE radial velocity measurements were combined with the photometry from the WASP and Euler Telescopes in a simultaneous fit using the Markov Chain Monte Carlo (MCMC) technique. The details of this process are described in Collier Cameron et al. (2007) and Pollacco et al. (2008). Four sets of solutions were used: with and without the main-sequence mass-radius constraint for both circular and floating eccentricity orbits. In all cases we have included a linear trend in the systemic velocity,
Table 2. Stellar parameters of WASP-34.

\begin{tabular}{ll}
\hline \hline Parameter & Value \\
\hline RA $(\mathrm{J} 2000.0)$ & $11 \mathrm{~h} 01 \mathrm{~m} 35.89 \mathrm{~s}$ \\
Dec $(\mathrm{J} 2000.0)$ & $-23^{\circ} 51^{\prime} 38.4^{\prime \prime}$ \\
$V \mathrm{mag}$ & 10.4 \\
Distance & $120 \pm 15 \mathrm{pc}$ \\
$T_{\text {eff }}$ & $5700 \pm 100 \mathrm{~K}$ \\
$\log g$ & $4.5 \pm 0.1$ \\
$\xi_{\mathrm{t}}$ & $0.9 \pm 0.1 \mathrm{~km} \mathrm{~s}^{-1}$ \\
$v \sin i$ & $1.4 \pm 0.6 \mathrm{~km} \mathrm{~s}^{-1}$ \\
{$[\mathrm{Fe} / \mathrm{H}]$} & $-0.02 \pm 0.10$ \\
{$[\mathrm{Si} / \mathrm{H}]$} & $+0.08 \pm 0.08$ \\
{$[\mathrm{Ca} / \mathrm{H}]$} & $+0.06 \pm 0.15$ \\
{$[\mathrm{Ti} / \mathrm{H}]$} & $+0.09 \pm 0.06$ \\
{$[\mathrm{Cr} / \mathrm{H}]$} & $-0.02 \pm 0.07$ \\
{$[\mathrm{Ni} / \mathrm{H}]$} & $+0.03 \pm 0.07$ \\
$\log A(\mathrm{Li})$ & $<0.8$ \\
$\mathrm{Spectral} \mathrm{Type}$ & $\mathrm{G} 5$ \\
$M_{\star}$ & $1.01 \pm 0.07 M_{\odot}$ \\
$R_{\star}$ & $0.93 \pm 0.12 R_{\odot}$ \\
\hline
\end{tabular}

Notes. The spectral type is that listed as G5 in the Yale Zone Catalogue (Schlesinger \& Barney 1943), which agrees with that implied from $T_{\text {eff }}$ (Gray 2008, Table B.1). $M_{\star}$ and $R_{\star}$ are estimated using the (Torres et al. 2010a) calibration.

Table 3. System parameters for WASP-34b.

\begin{tabular}{|c|c|}
\hline Parameter & Value \\
\hline Transit epoch (HJD), $T_{0}$ & $2454647.55358 \pm 0.00064$ \\
\hline Orbital period, $P$ & $4.3176782 \pm 0.0000045 \mathrm{~d}$ \\
\hline Transit duration, $T_{14}$ & $0.0876_{-0.0017}^{+0.0016} \mathrm{~d}$ \\
\hline Transit depth, $\left(R_{\mathrm{P}} / R_{*}\right)^{2}$ & $0.01261 \pm 0.00026$ \\
\hline Impact parameter, $b$ & $0.904_{-0.014}^{+0.017}$ \\
\hline Stellar reflex velocity, $K_{1}$ & $0.0721 \pm 0.0012 \mathrm{~km} \mathrm{~s}^{-1}$ \\
\hline Centre-of-mass velocity at time $T_{0}, \gamma$ & $49.9395 \pm 0.0064 \mathrm{~km} \mathrm{~s}^{-1}$ \\
\hline Drift in centre-of-mass velocity, $\frac{\mathrm{d} \gamma}{\mathrm{d} t}$ & $-54.8 \pm 4.0 \mathrm{~m} \mathrm{~s}^{-1} \mathrm{y}^{-1}$ \\
\hline Orbital separation, $a$ & $0.0524 \pm 0.0004 \mathrm{AU}$ \\
\hline Orbital inclination, $i$ & $85.2 \pm 0.2^{\circ}$ \\
\hline Orbital eccentricity, $e$ & $0.038 \pm 0.012$ \\
\hline Argument of periastron, $\omega$ & $-40.2_{-18.6}^{+23.2 \circ}$ \\
\hline$e \cos (\omega)$ & $+0.028 \pm 0.009$ \\
\hline$e \sin (\omega)$ & $-0.024 \pm 0.016$ \\
\hline Planet mass, $M_{\mathrm{P}}$ & $0.59 \pm 0.01 M_{\text {Jup }}$ \\
\hline Planet radius, $R_{\mathrm{P}}$ & $1.222_{-0.08}^{+0.11} R_{\text {Jup }}$ \\
\hline Planet surface gravity, $\log g_{\mathrm{P}}$ & $2.96_{-0.07}^{+0.05}$ \\
\hline Planet density, $\rho_{\mathrm{P}}$ & $0.34 \pm 0.08 \rho_{\text {Jup }}$ \\
\hline Planet equilibrium temperature, $T_{\mathrm{P}}$ & $1250 \pm 30 \mathrm{~K}$ \\
\hline
\end{tabular}

Notes. The planet equilibrium temperature, $T_{\mathrm{P}}$, assumes a Bond albedo of $A=0$ and even redistribution of heat around the planet.

$\frac{\mathrm{d} \gamma}{\mathrm{d} t}$, as a free parameter. Limb-darkening uses the four-coefficient model of Claret (2000) for the $r$-band, for both WASP and Euler photometry.

With the main-sequence constraint imposed but the eccentricity floating, a value of $e=0.038 \pm 0.012$ is found, which is significant at the $99.3 \%$ level (Lucy \& Sweeney 1971, Eq. (21)). The radial velocity measurements are well fitted by this orbital solution and the linear long-term drift (Fig. 5). However, a F-test 


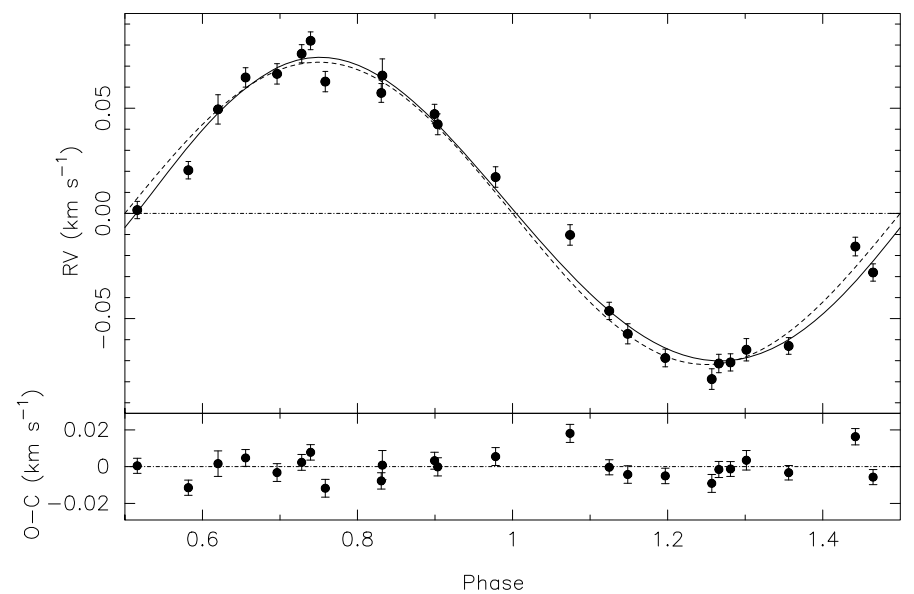

Fig. 5. Radial velocity measurements relative to the systemic-mass velocity including the long-term drift, $\frac{\mathrm{d} \gamma}{\mathrm{d} t}$, compared to the eccentric orbital solution for WASP-34b (solid line). The dashed-line is the corresponding circular orbital solution.

is a more reliable guide to the significance of the eccentricity (Lucy \& Sweeney 1971, Eq. (27)). The eccentric orbit fit has $\chi^{2}=57.4$, compared to $\chi^{2}=68.5$ for a circular orbit. We adopt the null hypothesis that the underlying orbit is truly circular but recognise that an eccentric fit to noisy and unevenly sampled data will always lead to a spurious improvement in $\chi^{2}$. The probability that an improvement in the fit could have arisen by chance with the addition of 2 fitting parameters, $e \cos (\omega)$ and $e \sin (\omega)$, is $17.1 \%$. While this is too high to confidently claim detection of eccentricity, it does not confirm that the orbit is truly circular. Hence, given there is some evidence for a non-circular orbit, we present the system parameters for the eccentric solution (Table 3). The impact parameter and transit depth suggest that the planet could be in a grazing transit.

Relaxing the main-sequence constraint yields a larger stellar radius $\left(1.20 \pm 0.09 R_{\odot}\right)$ and lower surface gravity $(\log g=4.3)$, which is marginally inconsistent with the spectroscopic $\log g$. The planetary radius increases to $1.82_{-0.48}^{+0.82} R_{\text {Jup }}$ and the impact parameter also rises to $0.98_{-0.06}^{+0.08}$. The planetary density now drops to $0.11_{-0.08}^{+0.15} \rho_{\text {Jup }}$, which would be similar to that found for WASP-17b (Anderson et al. 2010). While the fit to the transit lightcurves is only slightly different (Fig. 4), the uncertainties in the planetary parameters are now rather large, indicating that the near-grazing nature of the transit is poorly constrained by the available photometry. Consequently, we adopt the mainsequence-constrained eccentric solution.

\section{Discussion}

WASP-34b is a sub-Jupiter mass exoplanet transiting a G5 host star every 4.3177 days. A simultaneous fit to transit photometry and radial-velocity measurements gave a planetary mass of $0.59 \pm 0.01 M_{\text {Jup }}$ and radius of $1.22_{-0.08}^{+0.11} R_{\text {Jup }}$.

In many respects the WASP-34b system looks like transiting version of 51 Peg (Mayor \& Queloz 1995) with similar periods, separations, masses and host stars. Of the known transiting system, WASP-34b joins an increasing number of exoplanets with masses $\sim 0.5 M_{\text {Jup }}$ and radii $\sim 1.0 R_{\text {Jup }}$, such as WASP-22b (Maxted et al. 2010), WASP-25b (Enoch et al. 2010), HAT-P-24b (Kipping et al. 2010) and HAT-P-25b (Quinn et al. 2010). Of these systems, the similarity to WASP-22b is striking considering that it too has a linear trend in radial velocities due to a third-body in the system.

\subsection{Properties of the third body}

The significant linear trend in the radial velocities of $55 \pm$ $4 \mathrm{~m} \mathrm{~s}^{-1} \mathrm{y}^{-1}$, noted in Sect. 2.2, indicates the presence of a third body in the system with a long period. Using the constant acceleration method of Winn et al. (2009) the third body satisfies

$$
\frac{M_{c} \sin i_{c}}{a_{c}^{2}}=0.307 \pm 0.022
$$

where $M_{c}$ and $a_{c}$ are the mass (in $M_{\mathrm{Jup}}$ ) and orbital separation (in AU) of the third body. Given that the period of this outer body must be greater than at least twice the RV data baseline, $P_{c} \gtrsim 490$ days and, using Kepler's third law, $a_{c} \gtrsim 1.2$ AU. Hence $M_{c} \gtrsim 0.45 M_{\text {Jup }}$.

Using literature photometry we estimated the bolometric flux at the Earth to be $f_{\text {bol }}=1.97 \pm 0.10 \times 10^{-9} \mathrm{erg} \mathrm{s}^{-1} \mathrm{~cm}^{-2}$, which gives $T_{\text {eff }}=5740 \pm 140 \mathrm{~K}$ using the Infrared Flux Method (IRFM) (Blackwell \& Shallis 1977), which is in excellent agreement with the spectroscopic temperature determination. The modified IRFM method of Smalley (1993) suggests that a mainsequence companion would be cooler than $\sim 4000 \mathrm{~K}$ (M-type or later). There is a GALEX (Morrissey et al. 2007) near-UV flux measurement for WASP-34, and this is in in agreement with that predicted for a star of this temperature. This is no significant UV excess that could be attributed to a hot compact stellar companion, but this does not exclude the possibility of a cool white dwarf companion. Thus, we expect that the companion object is either a low mass star (M-type or cooler), cool white dwarf or another planet in a wide long-period orbit. Further radial velocity measurements are required to constrain the orbit.

\subsection{Grazing nature of the system}

The transit depth $\left(\left(R_{\mathrm{P}} / R_{*}\right)^{2}=0.0126\right)$ and high impact parameter ( $b=0.90$ ) suggest that WASP-34b could be the first transiting exoplanet known to undergo grazing transits. For a transit to be truly grazing, we require

$b+R_{\mathrm{P}} / R_{\star}>1$

and that this grazing criterion is significant compared to the observational uncertainties. For WASP-34b we find a value of $1.016_{-0.014}^{+0.017}$ for the grazing criterion. Hence, we might expect grazing transits, unlike the near-grazing transits of TrES-2 (O’Donovan 2006) and HAT-P-14b (Torres et al. 2010b), which have grazing criteria of $0.972 \pm 0.007$ and $0.968 \pm 0.022$, respectively. Figure 6 shows the 10000 MCMC posteriors for $R_{\mathrm{P}} / R_{\star}$ and $b$. A total of $89.6 \%$ of these satisfy the grazing criterion. If the grazing criterion were equal to unity, then we would expect $50 \%$ of the points to be higher and $50 \%$ to be lower than one. Using the odds ratio test (Kipping et al. 2010), we find a $82.8 \%$ probability $(1.4-\sigma)$ that the system is truly grazing. However, this is lower than a $3-\sigma$ value which would be a reasonable limit for detection of truly grazing system. Hence, we conclude that WASP-34b has near-grazing transits.

The eccentricity $(e)$ and argument of periastron $(\omega)$ are such that the occultation impact parameter will be

$b_{\text {occ }}=(0.953 \pm 0.031) b_{\text {tra }}=0.862 \pm 0.035$. 


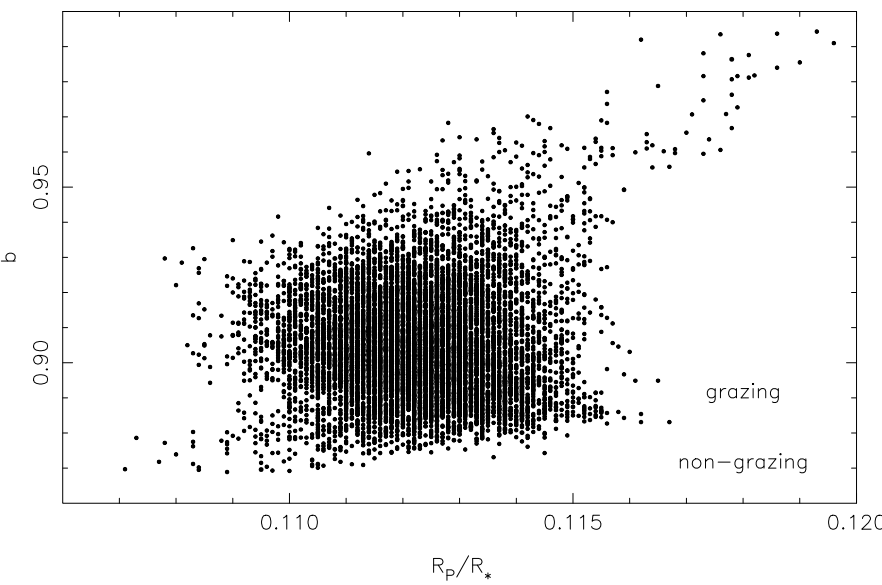

Fig. 6. Plot of the MCMC posteriors $b$ and $R_{\mathrm{P}} / R_{\star}$. The solid-line indicates the position of the stellar limb, i.e. $b+R_{\mathrm{P}} / R_{\star}=1$. A total of $89.6 \%$ of the points lie above the line and are grazing solutions.

Table 4. Effect of limb-darkening on the derived impact parameter, $b$, and transit depth, $\left(R_{\mathrm{P}} / R_{*}\right)^{2}$, for various filter bands.

\begin{tabular}{llllll}
\hline \hline Band & $b$ & $\left(R_{\mathrm{P}} / R_{*}\right)^{2}$ & $b+R_{\mathrm{P}} / R_{\star}$ & $\%(>1)$ & $P(>1)$ \\
\hline$v$ & 0.898 & 0.01309 & $1.012_{-0.013}^{+0.017}$ & 83.1 & 74.7 \\
$r$ & 0.904 & 0.01261 & $1.016_{-0.014}^{+0.017}$ & 89.6 & 82.8 \\
$i$ & 0.905 & 0.01213 & $1.015_{-0.012}^{+0.016}$ & 91.1 & 84.9 \\
$z$ & 0.907 & 0.01190 & $1.016_{-0.013}^{+0.016}$ & 90.3 & 83.8 \\
\hline
\end{tabular}

Notes. The grazing criterion, $b+R_{\mathrm{P}} / R_{\star}$, is given along with the percentage of MCMC posteriors that are grazing, $\%(>1)$, and the confidence values, $P(>1)$.

Hence, $b_{\text {occ }}+R_{\mathrm{P}} / R_{\star}=0.974 \pm 0.035$ with only $19.2 \%$ of the MCMC posteriors greater than 1. Therefore, the occultations of the planet behind the star are expected to be total. However, the uncertainties in the orbital elements are such that is a possibility that the occultations could be partial.

For transits with large $b$, the limb-darkening coefficients are extremely correlated to both $R_{\mathrm{P}} / R_{*}$ and $b$ (Kipping \& Bakos 2010). In order to investigate the effects of limb-darkening on the inferred grazing nature of the system, we re-ran our MCMC analysis using limb-darkening coefficients for different filter bands (Table 4). Compared to the $r$-band results, there are small but noticeable, differences. Hence, this heuristic demonstrates that there is a $75 \sim 85 \%$ probability that the transit is actually grazing.
The near-grazing nature of the system makes it sensitive to additional planets, via changes to transit duration and shape. Given that there is evidence for another object in the WASP-34 system, further monitoring of the transits is required.

Acknowledgements. WASP-South is hosted by the South African Astronomical Observatory and their support and assistance is gratefully acknowledged. M. Gillon acknowledges support from the Belgian Science Policy Office in the form of a Return Grant. We thank the referee, David Kipping, for his constructive and helpful comments on the original manuscript.

\section{References}

Anderson, D. R., Hellier, C., Gillon, M., et al. 2010, ApJ, 709, 159 Baranne, A., Queloz, D., Mayor, M., et al. 1996, A\&AS, 119, 373 Barnes, S. A. 2007, ApJ, 669, 1167

Blackwell, D. E., \& Shallis, M. J., 1977, MNRAS, 180, 177

Bruntt H., Bedding T. R., Quirion P.-O., et al., 2010, MNRAS, 405, 1907

Charbonneau, D., Allen, L. E., Megeath, S. T., Torres, G., \& Alonso, R. 2005, ApJ, 626, 523

Claret, A., 2000, A\&A, 363, 1081

Collier Cameron, A., Pollacco, D. L., Street, R. A., et al. 2006, MNRAS, 373, 799

Collier Cameron, A., Wilson, D. M., West, R. G., et al. 2007, MNRAS, 380, 1230

Enoch, B., Collier Cameron, A., Anderson, D. R., et al. 2010, MNRAS, in press [arXiv: 1009.5917]

Gillon, M., Smalley, B., Hebb, L., et al. 2009, A\&A, 496, 259

Gray, D. F., 2008, The observation and analysis of stellar photospheres, 3rd Ed. (Cambridge University Press), 507

Kipping, D. M. \& Bakos, G. A., 2010, ApJ, submitted [arXiv: 1006. 5680]

Kipping, D. M., Bakos, G. A., Hartman, J., et al. 2010, ApJ, 725, 2017

Lucy, L. B., \& Sweeney, M. A. 1971, AJ, 76, 544

Magain, P. 1984, A\&A, 134, 189

Maxted, P. F. L., Anderson, D. R., Gillon, M., et al. 2010, AJ, 140, 2007

Mayor, M., \& Queloz, D. 1995, Nature, 378, 355

Morrissey P., Conrow T., Barlow T. A. et al. 2007, ApJS, 173, 682

O’Donovan, F. T., Charbonneau, D., \& Mandushev, G. 2006, ApJ, 651, 61

Pepe, F., Mayor, M., Galland, F., et al. 2002, A\&A, 388, 632

Pollacco, D. L., Skillen, I., Collier Cameron, A., et al. 2006, PASP, 118, 1407

Pollacco, D. L., Skillen, I., Collier Cameron A., et al. 2008, MNRAS, 385, 1576

Queloz, D., Mayor, M., Weber, L., et al. 2000, A\&A, 354, 99

Queloz, D., Henry, G. W., Sivan, J. P., et al. 2001, A\&A, 379, 279

Quinn, S. N., Bakos, G. A., Hartman, J., et al. 2010, ApJ, submitted [arXiv: 1008.3565]

Schlesinger, F., \& Barney, I. 1943, Trans. Astron. Obs. Yale Univ., 14, 1

Sestito, P., \& Randich, S. 2005 A\&A, 442, 615

Smalley, B. 1993, MNRAS, 265, 1035

Smalley, B. 2005, Mem. Soc. Astron. Ital. Suppl., 8, 130

Southworth, J. 2009, MNRAS, 394, 272

Torres, G., Andersen, J., \& Giménez, A. 2010a, A\&ARv, 18, 67

Torres, G., Bakos, G. A., Hartman, J., et al. 2010b, ApJ, 715, 458

West R. G., Anderson D. R., Gillon M., et al. 2009, AJ, 137, 4834

Winn, J. N. 2009, IAU Symp., 253, 99

Winn, J. N., Johnson J. A., Albrecht S., et al. 2009, ApJ, 703, 99 